\title{
Comparison of Traditional Methods and Microwave Irradiation Method About Amylum/Acrylic Acid /Acrylamide Polymerization
}

Hao Ren, Zhen Niu, Juan Wang and Jing Ning

Additional information is available at the end of the chapter

http://dx.doi.org/10.5772/48610

\section{Introduction}

Based on previous work, the research in amylum grafted acrylic/acrylamide superabsorbent synthesis was made under traditional condition in this section. We synthetize binary grafted amylum superabsorbent and study its water absorption.

\subsection{Experiment}

\subsubsection{Reagents and instruments}

\subsubsection{Reagents}

\begin{tabular}{|l|l|}
\hline Soluble Amylum (Corn) & A.R. Tianjin Kemiou Chemical Reagents Development Center \\
\hline Wheat Amylum & F.G. Jinan Sanjia Sugar Co. LTD \\
\hline Cassava Amylum & F.G. Jinan Sanjia Sugar Co. LTD \\
\hline Potato Amylum & F.G. Qingzhou Zhengyi Seasoning Food Co. LTD \\
\hline Pachyrhizus Amylum & F.G. Qingzhou Zhengyi Seasoning Food Co. LTD \\
\hline Crylic Acid & C.P. Tianjin Kemiou Chemical Reagents Development Center \\
\hline Acrylamide & A.R. Tianjin Kemiou Chemical Reagents Development Center \\
\hline Potassium Hydroxide & A.R. Tianjin Kemiou Chemical Reagents Development Center \\
\hline Potassium Peroxydisulfate & A.R. Tianjin Sitong Chemical Plant \\
\hline N, N,-methylene biasacrylamide & C.P. Chemical Reagent of Traditional Chinese Medicine Co. LTD \\
\hline
\end{tabular}




\subsubsection{Instruments}

\begin{tabular}{|l|l|}
\hline 101-1 Constant Temperature Electrothermal Blowing Drying Oven & Shanghai Luda Experiment Instrument Co.LTD \\
\hline TG328B Lightning Analytical Balance & Shanghai Balance Instrument Plant \\
\hline JJ-1 Timing Electric Mixer & Jiangsu Zhongda Instrument Plant \\
\hline TENSOR27 FTIR & Germany \\
\hline Hitachi S-2500 SEM & Japanese Hitachi Co. LTD \\
\hline
\end{tabular}

\subsubsection{Experiment principle and preparation method}

1.1.2.1. Synthetic process flow of binary amylum grafted superabsorbent

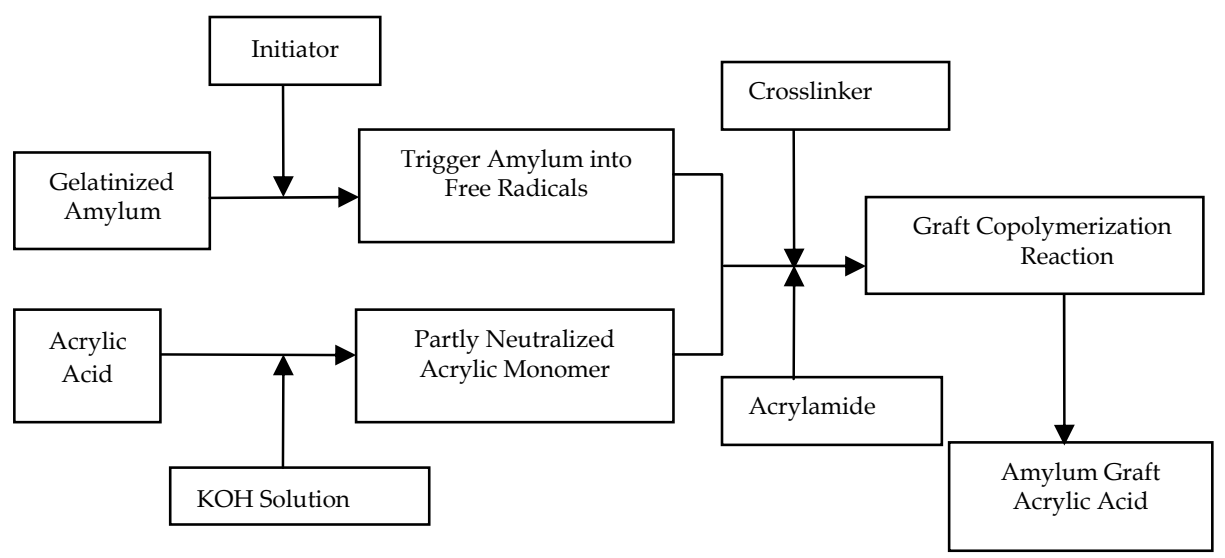

\section{Scheme 1.}

1.1.2.2. Major chemical reactions of binary grafted amylum superabsorbent synthesis

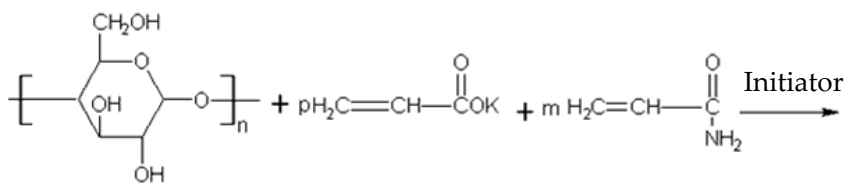

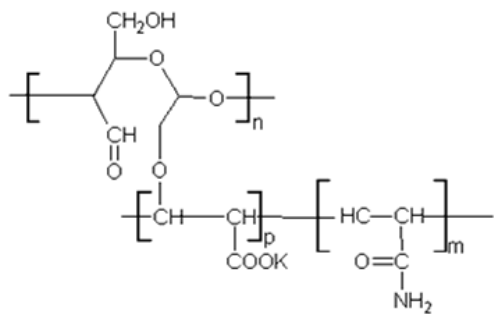

Scheme 2. 


\subsubsection{Process}

1. Taking $2.0 \mathrm{~g}$ soluble amylum into four-mouth bottle, joined with deionized water.

2. Pasting 20 minute in $50{ }^{\circ} \mathrm{C}$ water.

3. Changing dosage of acrylamide in basic Experiment conditions.

4. Designing orthogonal experiment and selecting orthogonal table L25 (56) of 6 factors and 5 levels according to the single factor experiment results, such as table 1 :

5. Adding right amount monomer, initiator and crosslinker according to orthogonal experiment requirements.

6. Beginning to react in experiment condition under the protection of nitrogen.

7. After the reaction, keeping it in $80^{\circ} \mathrm{C}$ drying oven to constant heavy.

\begin{tabular}{|c|c|c|c|c|c|c|}
\hline & Monomer/g & $\mathrm{T} /{ }^{\circ} \mathrm{C}$ & $\mathrm{t} / \mathrm{h}$ & Neutralization degree/\% & Initiator/g & Crosslinker/g \\
\hline 1 & 10 & 30 & 1.0 & 86 & 0.01 & 0.0009 \\
\hline 2 & 11 & 40 & 1.5 & 88 & 0.02 & 0.0011 \\
\hline 3 & 12 & 50 & 2.0 & 90 & 0.03 & 0.0013 \\
\hline 4 & 13 & 60 & 2.5 & 92 & 0.04 & 0.0015 \\
\hline 5 & 14 & 70 & 3.0 & 94 & 0.05 & 0.0017 \\
\hline
\end{tabular}

Table 1. Orthogonal Experiment level Factor

\subsubsection{Test method}

\subsubsection{Test of bibulous rate}

There is a variety of methods testing which can test the water absorption of superabsorbent, such as low method, paper bag method, natural filtering method, sieve net method, and so on. There are many differences between data from various test methods and it is hard to compare different data of bibulous rate from various documents.

The sieve net method in this research has been widely used by many researchers. The procedures as follows: taking certain quality $\left(\mathrm{m}_{1}\right)$ product into the beaker, adding excessive deionized water and physiological saline, Standing before swelling completely, then screening and filtering the excessive water by 100 mesh screen cloth and adsorbing surface water of resin by paper absorbent. Then weighing $\left(\mathrm{m}_{2}\right)$ it, calculating bibulous rate according to equation 1(Hongke Tang\& Qi Chen, 2007):

$$
\mathrm{Q}=\left(\mathrm{m}_{2}-\mathrm{m}_{1}\right) / \mathrm{m}_{1} \times 100 \% \ldots \ldots \ldots \ldots
$$

\subsubsection{Structure characterization of bibulous resin}

1. Infrared Spectrometry

Making corn amylum and absorbent resin dried fully and detecting it using infrared spectrophotometric method.

\section{Scanning Electron Microscopy}

The exterior characteristics were observed by the scanning electron microscopy (SEM) on the $1 \mathrm{~cm}^{2}$ resin. 


\subsection{Results and discussions}

\subsubsection{Ratio of acrylamide and acrylic acid}

The result of Figure 1 shows that concentration of acrylamide in a certain range can improve the bibulous rate of resin. Different ionization degree affected water absorption of resin in the composition of monomer. In acrylamide molecule, $-\mathrm{CONH}_{2}$ group is nonionic group and its dissociation degree in the water is small. So the effect of ion on it is small. The existence of $-\mathrm{CONH}_{2}$ can improve water absorption because of synergy of groups. But if the dosage was too much, water absorption reduced because the $-\mathrm{CONH}_{2}$ is less hydrophilic than COONa.

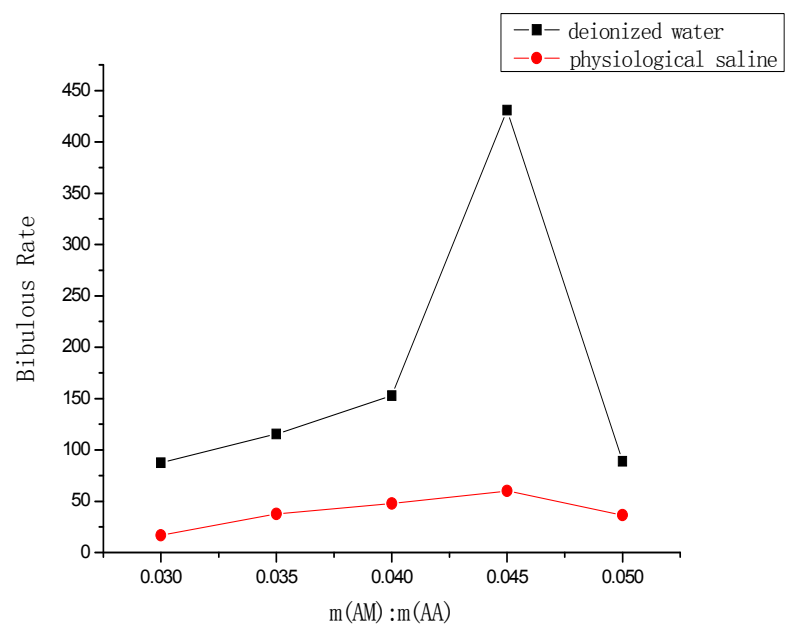

Figure 1. Effect of $\mathrm{m}(\mathrm{AM}): \mathrm{m}(\mathrm{AA})$ 


\subsubsection{Orthogonal Analysis}

\subsubsection{Analysis of the Orthogonal Experiment (Table 2)}

\begin{tabular}{|c|c|c|c|c|c|c|c|c|}
\hline & Monomer & $\mathrm{T}$ & $\mathrm{t}$ & $\begin{array}{c}\text { Neutralization } \\
\text { Degree }\end{array}$ & Initiator & Crosslinker & $\begin{array}{c}\text { Water } \\
\text { Absorption }\end{array}$ & $\mathrm{Q}$ \\
\hline 1 & 1 & 1 & 1 & 1 & 1 & 1 & 82.67 & 24.6 \\
\hline 2 & 1 & 2 & 2 & 2 & 2 & 2 & 402.66 & 31.02 \\
\hline 3 & 1 & 3 & 3 & 3 & 3 & 3 & 462.92 & 62.24 \\
\hline 4 & 1 & 4 & 4 & 4 & 4 & 4 & 246.49 & 34.89 \\
\hline 5 & 1 & 5 & 5 & 5 & 5 & 5 & 36.66 & 2.65 \\
\hline 6 & 2 & 1 & 2 & 3 & 4 & 5 & 388.02 & 65.66 \\
\hline 7 & 2 & 2 & 3 & 4 & 5 & 1 & 232.99 & 36.2 \\
\hline 8 & 2 & 3 & 4 & 5 & 1 & 2 & 220.43 & 27.46 \\
\hline 9 & 2 & 4 & 5 & 1 & 2 & 3 & 465.22 & 23.57 \\
\hline 10 & 2 & 5 & 1 & 2 & 3 & 4 & 416.33 & 43.11 \\
\hline 11 & 3 & 1 & 3 & 5 & 2 & 4 & 699.99 & 70.12 \\
\hline 12 & 3 & 2 & 4 & 1 & 3 & 5 & 457.62 & 65.71 \\
\hline 13 & 3 & 3 & 5 & 2 & 4 & 1 & 308.27 & 27.02 \\
\hline 14 & 3 & 4 & 1 & 3 & 5 & 2 & 296.39 & 62.61 \\
\hline 15 & 3 & 5 & 2 & 4 & 1 & 3 & 410.54 & 21.43 \\
\hline 16 & 4 & 1 & 4 & 2 & 5 & 3 & 328.72 & 30.42 \\
\hline 17 & 4 & 2 & 5 & 3 & 1 & 4 & 491.14 & 32.84 \\
\hline 18 & 4 & 3 & 1 & 4 & 2 & 5 & 476.81 & 48.12 \\
\hline 19 & 4 & 4 & 2 & 5 & 3 & 1 & 41.27 & 24.31 \\
\hline 20 & 4 & 5 & 3 & 1 & 4 & 2 & 197.49 & 29.88 \\
\hline 21 & 5 & 1 & 5 & 4 & 3 & 2 & 285.61 & 31.44 \\
\hline 22 & 5 & 2 & 1 & 5 & 4 & 3 & 313.19 & 47.79 \\
\hline 23 & 5 & 3 & 2 & 1 & 5 & 4 & 355.47 & 55.76 \\
\hline 24 & 5 & 4 & 3 & 2 & 1 & 5 & 335.48 & 54.91 \\
\hline 25 & 5 & 5 & 4 & 3 & 2 & 1 & 205.26 & 30.86 \\
\hline K1 & 266.28 & 357 & 317.08 & 311.69 & 308.05 & 174.09 & & \\
\hline K2 & 344.6 & 379.52 & 319.96 & 358.29 & 449.94 & 280.52 & & \\
\hline $\mathrm{K} 3$ & 434.56 & 364.78 & 385.77 & 368.75 & 332.75 & 396.12 & & \\
\hline K4 & 307.09 & 296.97 & 311.7 & 330.49 & 290.69 & 441.88 & & \\
\hline K5 & 299 & 253.26 & 317.38 & 262.31 & 250.05 & 329.92 & & \\
\hline $\mathrm{R}$ & 168.28 & 126.26 & 74.07 & 106.44 & 199.89 & 267.79 & & \\
\hline K1' & 31.08 & 44.45 & 45.25 & 39.9 & 32.25 & 28.6 & & \\
\hline K2' & 39.2 & 42.71 & 39.63 & 37.3 & 40.74 & 36.48 & & \\
\hline K3' & 49.38 & 44.12 & 50.65 & 50.84 & 45.36 & 37.09 & & \\
\hline K4' & 33.11 & 40.06 & 37.87 & 34.42 & 41.05 & 45.54 & & \\
\hline K5' & 44.15 & 25.59 & 23.5 & 34.46 & 37.53 & 47.41 & & \\
\hline $\mathrm{R}^{\prime}$ & 16.2 & 18.86 & 27.15 & 16.42 & 13.11 & 18.81 & & \\
\hline
\end{tabular}

Table 2. Analysis of the orthogonal experiment 


\subsubsection{Analysis of range}

Through the orthogonal experiment analysis, the influence of six factors of water absorption from big to small in order is: crosslinker concentration $>$ initiator concentration $>$ monomer concentration $>$ temperature $>$ neutralization degree $>$ reaction time. The best combination is $\mathrm{A}_{3} \mathrm{~B}_{2} \mathrm{C}_{3} \mathrm{D}_{3} \mathrm{E}_{2} \mathrm{~F}_{4}$.

\subsubsection{Analysis of monomer concentration}

Bibulous rate was highest when dosage of acrylic acid and acrylamide was $12.0 \mathrm{~g}$, bibulous rate reduced with the increase of monomer dosage after $12.0 \mathrm{~g}$ (Figure 2). Monomer in the certain range increased and the generation of copolymer increased. The better crosslinking, the better the water absorption will be.

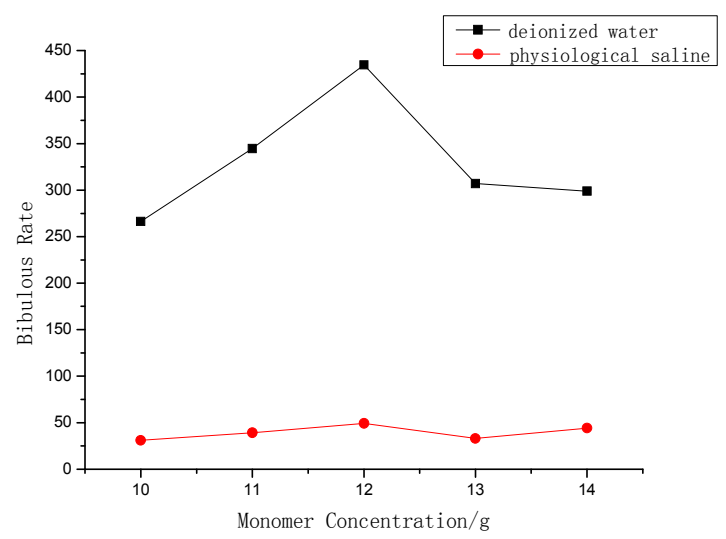

Figure 2. Effect of monomer concentration

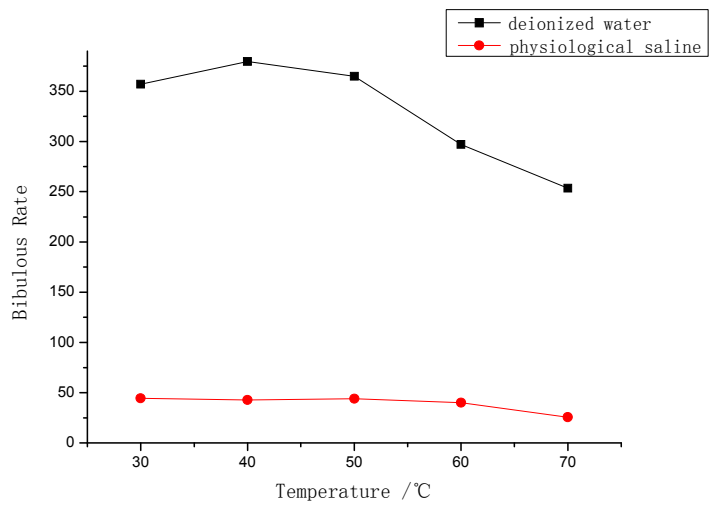

Figure 3. Effect of reaction temperature 


\subsubsection{Analysis of reaction temperature}

We could see the best reaction temperature was $40^{\circ} \mathrm{C}$ from Figure 3 . The temperature which is less than it was too low to facilitate the decomposition of initiator, as a result less free activity radicals were produced for the increase of the grafted rate and longer polymer chain reduced the bibulous rate of product. But when reaction temperature was more than $40^{\circ} \mathrm{C}$, as chain transfer and chain termination speeded up, the grafted rate and bibulous rate (Yan Huang \& Jiarui Shen, 1995) reduced.

\subsubsection{Analysis of reaction time}

Seeing from the Figure 4, the advisable polymerization time is $2 \mathrm{~h}$. If the reaction time was too long, bibulous rate of resin was slightly lower, perhaps due to the reason that crosslinking density increased. When polymerization time was brief, the polymerization was incomplete and molecular mass of the product was low (Ge Y X \& Zhang B Z, 2006), Network space became small after swelling, so the bibulous rate of resin reduced.

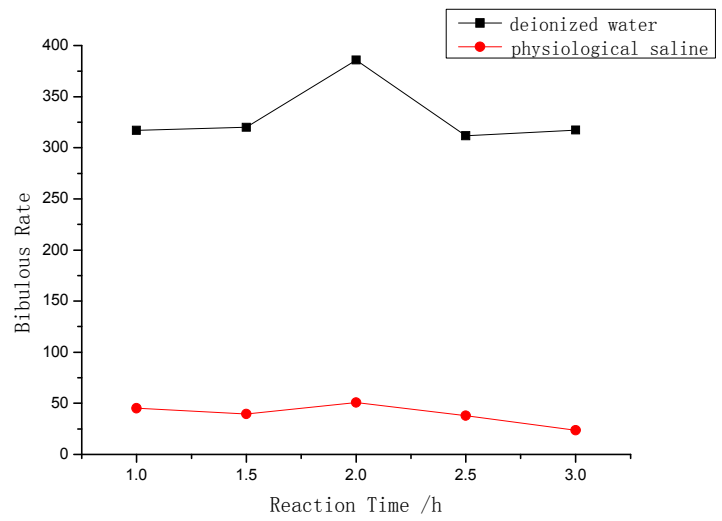

Figure 4. Effect of reaction time

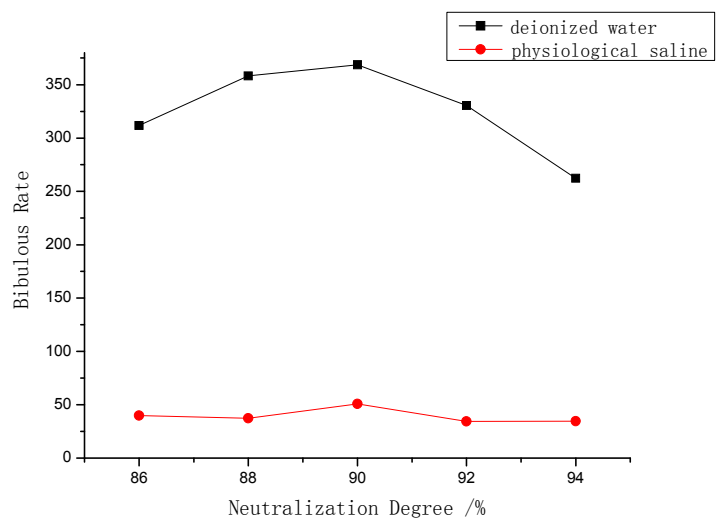

Figure 5. Effect of neutralization degree 


\subsubsection{Analysis of neutralization degree}

Figure 5 indicates that the neutralization degree has great influence on the water absorption, and the bibulous rate is higher when the neutralization degree is $90 \%$. Increasing the neutralization degree is increasing the concentration of the -COOK ion in the reaction system in essence. The dissociation ability of - $\mathrm{COOK}$ is far greater than $-\mathrm{COOH}$ in water, so the more -COOK led to more ionized -COO-. The increase of carboxylate ions in the lattice chain makes chain stretched and the repulsion of chains becomes strong, so the expansion of network polymerization increases, so does bibulous rate. However, with the increase of neutralization degree, the shielding effect of $\mathrm{K}^{+}$on carboxylate ion is gradually obvious in the system. And this kind of shielding effect will weaken repulsion between the chain and chain or adjacent carboxylate ions in the same chain, making the network expansion force reduced. Though neutralization degree is oversize, bibulous rate will reduce (Zhou M \& Lin J M, 2000).

\subsubsection{Analysis of initiator concentration}

We could see that bibulous rate increased, along with the increase of the initiator concentration when concentration was less than $0.02 \mathrm{~g}$, bibulous rate decreased instead when concentration was more than $0.02 \mathrm{~g}$ in Figure 6.

\subsubsection{Analysis of crosslinker concentration}

Figure 7 shows that bibulous rate was the highest when crosslinker concentration was $0.0015 \mathrm{~g}$. That is because the crosslinking density is low, water molecules that easily seep into the resin make resin inflated, further closed to water to become gel and then appear the state with high bibulous. The longer the chain and the bigger crosslinking structure are, the larger swelling volume and the more water absorption are. But crosslinking density can't be too low, or you will make the product soluble in water. It is better to be in the lowest crosslinking degree under the condition of insolubility in water.

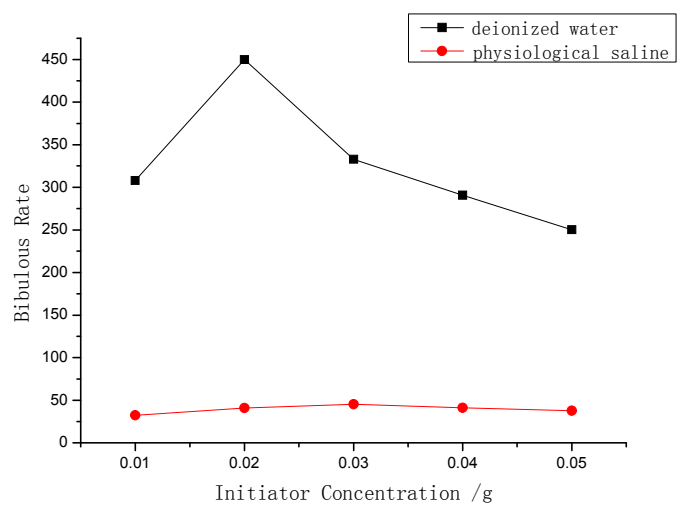

Figure 6. Effect of initiator concentration 


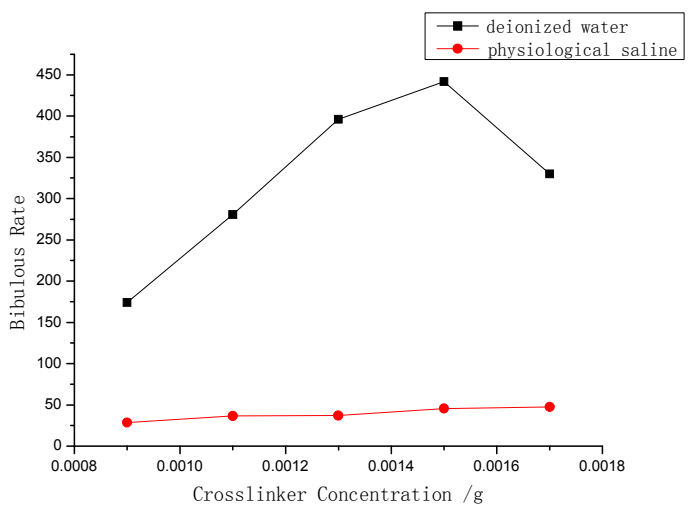

Figure 7. Effect of crosslinker concentration

\subsubsection{Amylum Choice}

It was clear that the water absorption of resin which was synthetized by corn amylum was much better than other kinds of amylum (Figure 8).

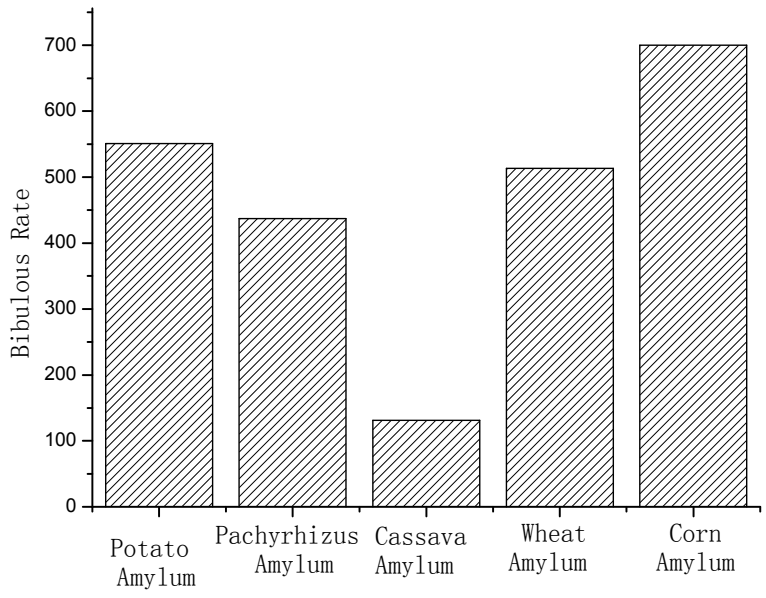

Figure 8. Effect of amylum 


\subsubsection{FTIR and SEM}

\subsubsection{Spectrum of FTIR}

The infrared spectra (Figure 9) analysis shows that amylum and its grafted products appear -OH characteristic peak in $3490 \mathrm{~cm}^{-1}$.Synthetic resin retained the amylum characteristic peak, and telescopic vibration absorption spectrum of $\mathrm{C}=\mathrm{O}$ of carboxylic acid and acylamino appeared in $1571 \mathrm{~cm}^{-1}$ and $1714 \mathrm{~cm}^{-1}$ which are the characteristic absorption peaks of grafted acrylic acid and acrylamide. The telescopic vibration absorption spectrum of $\mathrm{C}-\mathrm{N}$ of amide in $1407 \mathrm{~cm}^{-1}$ is the characteristic absorption peak of grafted acrylamide. All of these are the proof of successful grafting.

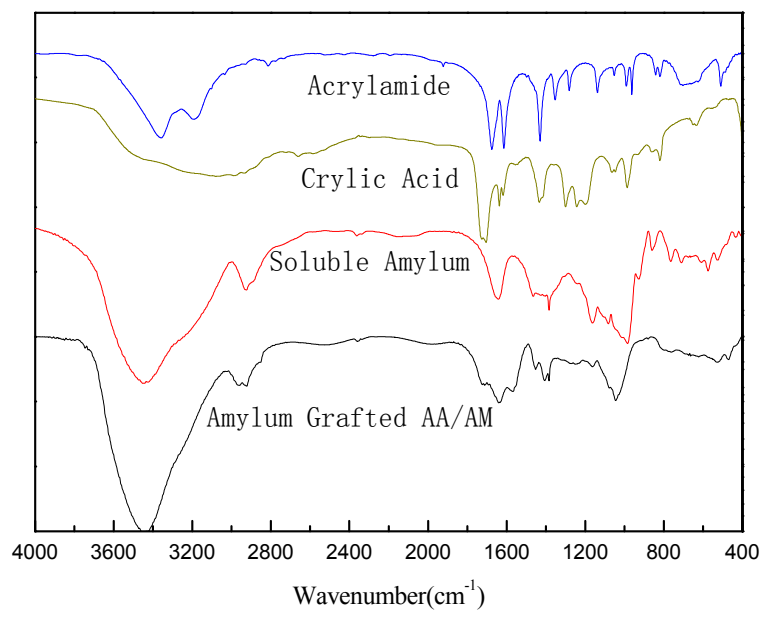

Figure 9. Spectrum of FTIR

\subsubsection{SEM micrograph of superabsorbent}

Grafting resin is network macromolecule formed by the skeleton polymers and branch polymers. Amylum, which belongs to hydrophilic semi-rigid bonds, makes polymer net into big space volume. This will form a developed hydrophilic network structure with a hole when amylum branch and grafting acrylic acid potassium, acrylamide branch chain interlace with each other. 


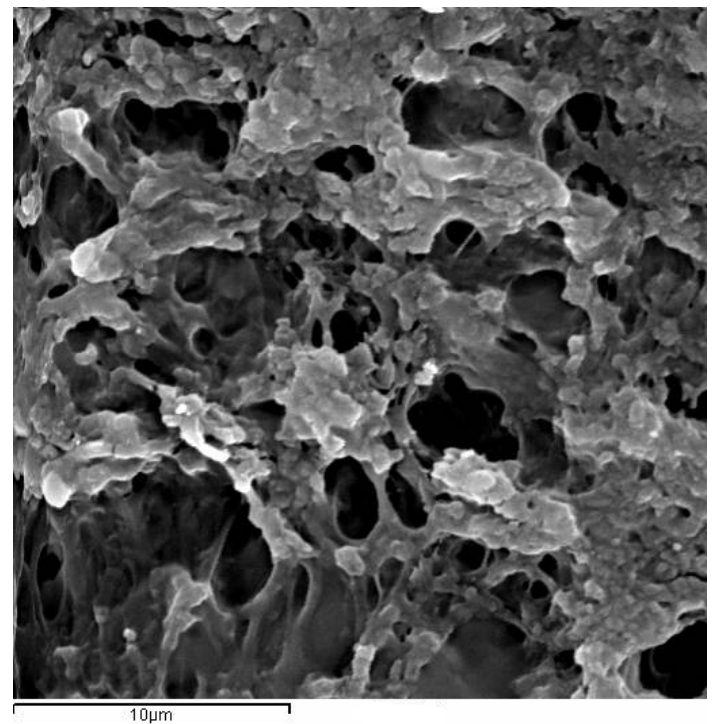

Figure 10. SEM micrograph of superabsorbent $(\times 2000)$

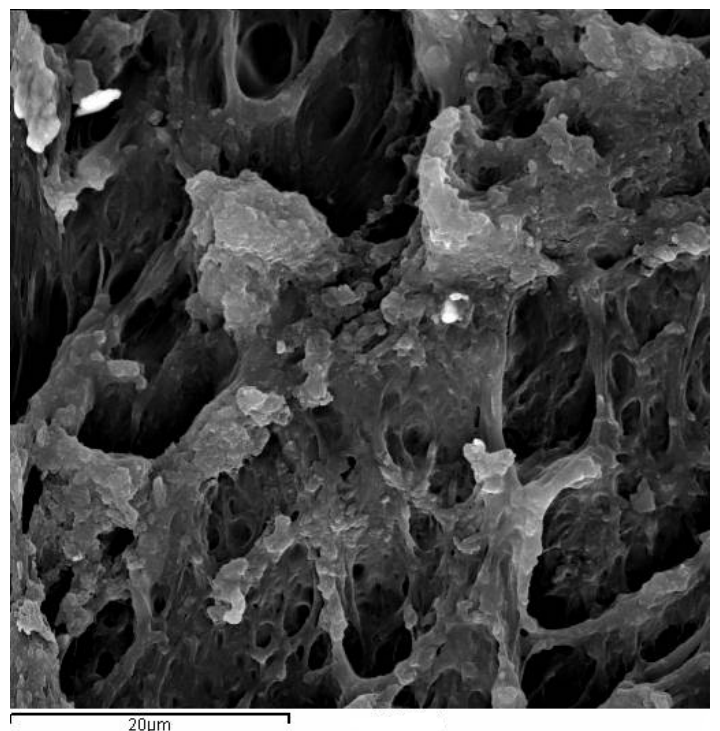

Figure 11. SEM micrograph of superabsorbent $(\times 4000)$

Figure 10 and Figure 11 are respectively the resin SEM micrograph pictures magnified 2000 times and 4000 times. We can see that the products form a three-dimensional network structure. The network structure is obviously dense and even. That is why binary grafted amylum superabsorbent has better water absorption than monobasic grafted amylum superabsorbent. 


\subsection{Summary}

This chapter uses aqueous solution method for the study of amylum grafted acrylic/acrylamide superabsorbent resin. Through the exploration experiment, we make sure that choosing corn amylum as experiment material, potassium peroxydisulfate as the initiator and nitrogen gas as reaction protection gas. Known from the experiment result, amylum category, monomer concentration, initiator concentration, neutralization degree, polymerization temperature, polymerization time and crosslinker concentration and so on all have certain effect on water absorption.

Under the condition of determining gelatinization type and certain amylum, the chapter works out the best reaction condition of the preparation of corn amylum grafted acrylic/acrylamide superabsorbent resin .when monomer concentration is $12.0 \mathrm{~g}$, reaction temperature is $30^{\circ} \mathrm{C}$, reaction time is $2 \mathrm{~h}$, acrylic acid neutralization degree is $94.0 \%$, initiator concentration is $0.02 \mathrm{~g}$ and crosslinker concentration is $0.0015 \mathrm{~g}$, a better result can be obtained. The absorbent ratio of water is up to 699 times and the absorbent ratio of physiological saline is up to 70 times.

\section{Synthesis of super-absorbent resin with the microwave method}

\subsection{Introduction}

\subsubsection{Introduction to Microwave Chemistry Mechanism}

Microwave chemistry is a new cross-disciplinary study (Qinhan Jin, 1999) of chemical application. It is developed on the base of research on object property and interaction characteristics in the microwave field. It can be said that the microwave chemistry is a science, according to the electromagnetic field and the electromagnetic wave theory, the dielectric physical theory, condensed matter physics theory, plasma physics theory, material structure theory and chemical theory, using the modern microwave technology to research the physical and chemical behavior of material under microwave field.

Microwave (Fan Shouyong, 2003) is electromagnetic wave whose frequency is about 300 $\mathrm{MHz}-300 \mathrm{GHz}$, namely the wavelength within the scope of the $100 \mathrm{~cm}^{-1} \mathrm{~mm}$. It is located between infrared radiation (light wave) of the electromagnetic spectrum and radio waves. Among it, the microwave band of $1-25 \mathrm{~cm}$ wavelength is specially used in radar, and the rest are used in telecommunications transmission. In order to prevent the interference of microwave power on radio communications, broadcast, television and radar, the microwave power frequency band of industry, scientific research, medicine, the household and civil has been ruled internationally. At present, the microwave technology has already been widely used in industrial and agricultural production, scientific research, medicine and so on.

Different from traditional Heating Method which heats conduction from the surface to the inside by thermal radiation, microwave heating is body heat caused by material dielectric loss in the electromagnetic field and at the same time electromagnetic energy transfer into heat energy. Due to the high efficiency and uniform heating characteristic of microwave 
heating, microwave heating can be applied to chemical reaction, influencing the reaction rate. Microwave, mainly includes microwave chemistry effect on condensed matter material, effect various chemical reactions by directly reacting with different kinds of chemical systems that are normally within the scope of microwave chemistry. The microwave chemistry also includes microwave effect on gaseous materials and microwave plasma chemistry.

\subsubsection{Microwave}

Gedye has found that microwave can promote organic reactions since 1986, microwave technology has been widely used in many organic reactions. A large number of Experiment results show that compared with traditional method, organic reactions under the action of microwave can speed up several times, several dozens of times even hundreds of times, and the biggest can be up to 1240 times. Now there are two different views about the reason why microwave can accelerate organic reactions in academia (Qinhan Jin, 1999).

Some academics thought that microwave heating is internal heating. It is fast, uniform and has no temperature gradient, hysteresis effect and many other characteristics. But microwave heating applied in chemical reactions is just a heating method, same as the traditional heating method. In a particular reaction, the kinetic will not change under the condition of invariable reactants, catalyst and product, without relation of heating method. They think that the microwave of $2450 \mathrm{MHz}$ frequency used for chemical reaction belonged to the non-ionized radiation. When resonance with molecular chemical bond, it could not cause chemical bond fractured and make molecules promoted to higher rotational level and vibrative level. Therefore, the reason why microwave can accelerate organic reactions was attributed to the selective heating of the polarity organic, namely microwave radiation thermal effect.

Others believe that the effect of microwave on chemical reaction was extremely complex. On the one hand, the reactant molecule absorbed the energy of microwave, improved the movement speed of molecular. So the molecular motion became desultorily, which led to the increase in entropy. On the other hand the effect of microwave on polarity molecules force the molecular to move by the way which are under the function of the electromagnetic field and changing $2.45 \times 10^{3}$ times per second. Due to the decrease of the entropy, the effect mechanism of microwave on chemical reaction was not just described by microwave radiation thermal effect. Besides heating effect, microwave also had a kind of non-thermal effects that were not caused by the temperature. Organic reactions under the action of microwave changed the reaction kinetics and reduced the reaction activation energy.

Microwave application in organic synthesis has been more than 10 years, however, research on microwave accelerating mechanism is a new field, still in the initial stage now. Some reaction results are lack of experiment argumentations and many experiment phenomenon need to be explained more comprehensively, meticulously and systematically.

At present, the microwave research mainly focus on the following three aspects on organic synthetic chemistry: 
1. Further improvement and establishment of new technology on microwave synthesis reaction technology.

2. Application and law of microwave organic synthesis reaction.

3. Systematic research of microwave chemistry theory.

\subsubsection{Application of microwave chemistry}

As is known to all, the traditional heating gradually heat conduction from the surface to the inside through the thermal radiation. In order to avoid excessive temperature gradient, heating speed often can't be too fast. What's more, it can't heat the different components of mixed materials in the same response device selectively.

Compared with traditional heating method, microwave heating has following characteristics ${ }^{[17]}$ :

1. It can heat material from outer to inner gradually, and heating rate is quick.

2. High thermal efficiency and low energy consumption.

3. It has no hysteresis effect and it is easy to realize heating equably, also with low temperature gradient.

4. Heat each component of mixed materials selectively and realize automation control easily.

5. It can also promote endothermic reaction and exothermic reaction and has catalytic effect on some chemical reactions.

6. Reduce some chemical reaction temperature.

7. Make some high polymer materials to have ideal performance (Du T S \& Kang S Z, 2000).

Microwave technology with such characteristics was widely used in organic synthesis, chemical analysis, food processing, medical science and many other fields.

The application of microwave organic synthesis technology has begun in the 1980s.After Gedye and mates found that applying microwave heating technology in organic synthesis of small molecules could significantly improve the reaction rate, using microwave technology to promote organic reactions became the focus of attentions. Nowadays the microwave heating organic synthesis reactions are consist of the alkylation reaction, esterification reaction, substitution and elimination reaction, sulphonation reaction, olefins addition reaction, condensation reaction, rearrangement reaction, pericyclic reaction, organic metal reaction and so on. The research shows that, microwave technology applied in organic synthesis not only can improve the reaction speed and shorten the reaction time, but also can help to have easy operation, high yield, pure product and other advantages (Wangxi Zhang, 2004).

In addition, microwave heating technology plays a support role on the synthesis and processing of polymer compound with high viscosity and low thermal conductivity, also the modification and curing of natural polymer. 


\subsection{Experiment}

\subsubsection{Reagents and instruments}

\subsubsection{Reagents}

\begin{tabular}{|l|l|}
\hline Soluble Amylum (Corn) & A.R. Tianjin Kemiou Chemical Reagents Development Center \\
\hline Crylic Acid & C.P. Tianjin Kemiou Chemical Reagents Development Center \\
\hline Potassium Hydroxide & A.R. Tianjin Kemiou Chemical Reagents Development Center \\
\hline Potassium Peroxydisulfate & A.R. Tianjin Sitong Chemical Plant \\
\hline $\mathrm{N}, \mathrm{N},-$-methylene biasacrylamide & C.P. Chemical Reagent of Traditional Chinese Medicine Co. LTD \\
\hline
\end{tabular}

\subsubsection{Instruments}

\begin{tabular}{|l|l|}
\hline MAS-I Microwave Reactor & $\begin{array}{l}\text { New Instrument Microwave Chemical } \\
\text { Technology Co. LTD }\end{array}$ \\
\hline $\begin{array}{l}\text { 101-1 Constant Temperature Electrothermal Blowing } \\
\text { Drying Oven }\end{array}$ & Shanghai Luda Experiment Instrument Co.LTD \\
\hline TG328B Lightning Analytical Balance & Shanghai Balance Instrument Plant \\
\hline JJ-1 Timing Electric Mixer & Jiangsu Zhongda Instrument Plant \\
\hline TENSOR27 FTIR & Germany \\
\hline Hitachi S-2500 SEM & Japanese Hitachi Co. LTD \\
\hline
\end{tabular}

\subsubsection{Experiment procedure}

1. Take $2 \mathrm{~g}$ corn amylum into four-mouth bottle and add certain deionized water. Put the bottle into the microwave synthesis device after intensive mixing and connect it with condenser pipe and thermometer, stirring 20 minute under $50{ }^{\circ} \mathrm{C}$, gelatinization with the microwave power in $800 \mathrm{~W}$.

2. After the viscosity, cool it naturally to room temperature. The acrylic acid and acrylamide with a certain neutralization degree (which were neutralized by $\mathrm{KOH}$ ), initiator and crosslinker were added.

3. Continue radiating in certain microwave power and temperature for some time.

4. When reaction was over, dismantle device, move the product to the small dish and dry it to constant weight.

5. Crush the product and do the performance measurement and structure characterization.

\subsubsection{Test method}

\subsubsection{Test of bibulous rate}

There are variety of methods to test the water absorption of superabsorbent, such as low method, paper bag method, natural filtering method, sieve net method and etc. The data from various test methods have big differences and it is hard to compare different data of bibulous rate from various documents. 
The sieve net method is used widely by many researchers, which is known that take certain quality $\left(\mathrm{m}_{1}\right)$ product into the beaker and add excessive deionized water and physiological saline. Standing before swelling completely, then screen and filter the excessive water by 100 mesh screen cloth and adsorb surface water of resin using paper absorbent. Then weigh $\left(\mathrm{m}_{2}\right)$ it and calculate bibulous rate.

\subsubsection{Structure Characterization of Bibulous Resin}

1. Infrared Spectrometry

Making corn amylum and absorbent resin dried fully, potassium bromide was used for tabletting.

\section{Scanning Electron Microscope Method}

The exterior characteristics was selected and observed on the $1 \mathrm{~cm}^{2}$ resin by the scanning electron microscopy (SEM).

\subsection{Results and discussions}

\subsubsection{Polymerization temperature}

Reaction temperature has a certain effect on superabsorbent resin. When the temperature is lower, polymerization speed is slower, some resin crosslink incompletely and bibulous rate declines. When the temperature is too high, on the one hand initiator is beneficial to initiate polymerization. On the other hand chain transfer and chain termination speed up so that soluble part of the superabsorbent resin increases and bibulous rate declines. In this paper, we made a research on the effect of reaction temperature on water absorption under the fixed other conditions.

The study found that bibulous rate reached maximum when the reaction temperature was $35^{\circ} \mathrm{C}$ (Figure 12).

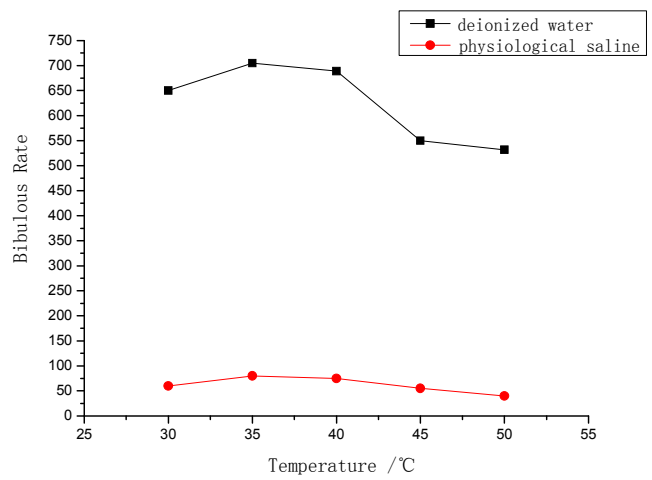

Figure 12. Effect of reaction temperature 


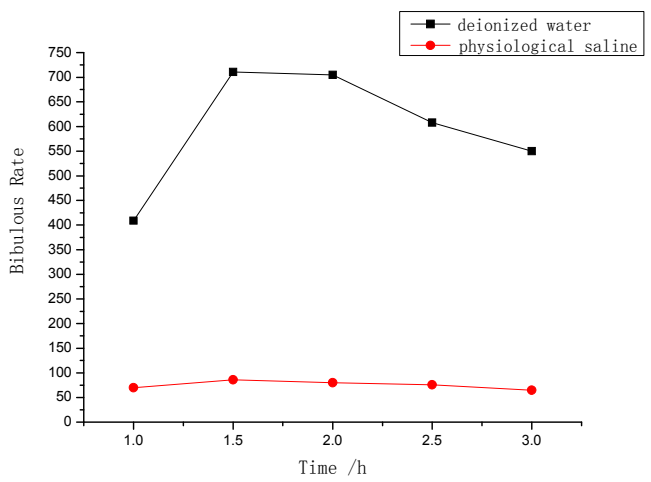

Figure 13. Effect of reaction time

\subsubsection{Polymerization time}

The reaction speed could be accelerated in the microwave condition, and reaction free radicals mechanism did not change. Microwave radiation made polymerization reaction speed increased, microwave inducing made reaction period shortened. Figure 13 is curve of the bibulous rate of synthetic resin with the change of microwave radiation time. The Experiment result showed that the microwave radiation could make the grafting copolymerization reaction speed greatly improved, reaction cycle shortened and water absorption performance was also improved on the whole. This may be caused by two reasons. One was the even synchronous heating characteristic of microwave, makinge the initiator and monomer of the system synchronously triggered, simultaneously reacted. And microwave heating rate was so quick that it can reach the needed temperature of system quickly. The other one was by means of microwave radiation response, may be "nonthermal effect" which made the water absorption performance improved. But with the increase of microwave reaction time, the bibulous rate of superabsorbent resin decreased. The reason may be that the radiation time was too long and resin crosslinking degree increased, so bibulous rate of synthetic resin was down.

\subsubsection{FTIR and SEM}

\subsubsection{Spectrum of FTIR}

The infrared spectra (Figure 14) analysis shows that amylum and its grafted products appear -OH characteristic peak in $3490 \mathrm{~cm}^{-1}$. Synthetic resin retained the amylum characteristic peak, and telescopic vibration absorption spectrum of $\mathrm{C}=\mathrm{O}$ of carboxylic acid and acylamino appeared in $1574 \mathrm{~cm}^{-1}$ and $1722 \mathrm{~cm}^{-1}$, which are the characteristic absorption peaks of grafted acrylic acid and acrylamide. The telescopic vibration absorption spectrum of C-N of amide in $1405 \mathrm{~cm}^{-1}$, is the characteristic absorption peak of grafted acrylamide. All of these prove the success of this experiment. 


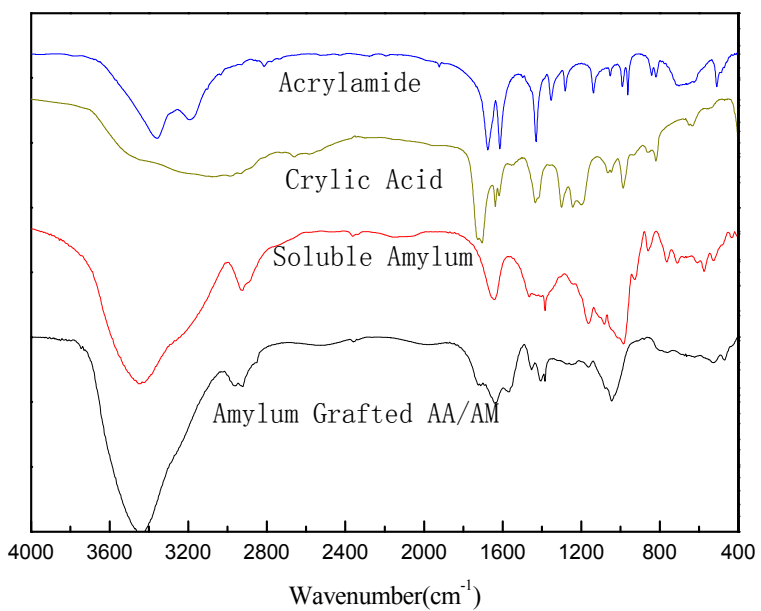

Figure 14. Spectrum of FTIR

\subsubsection{Scanning picture}

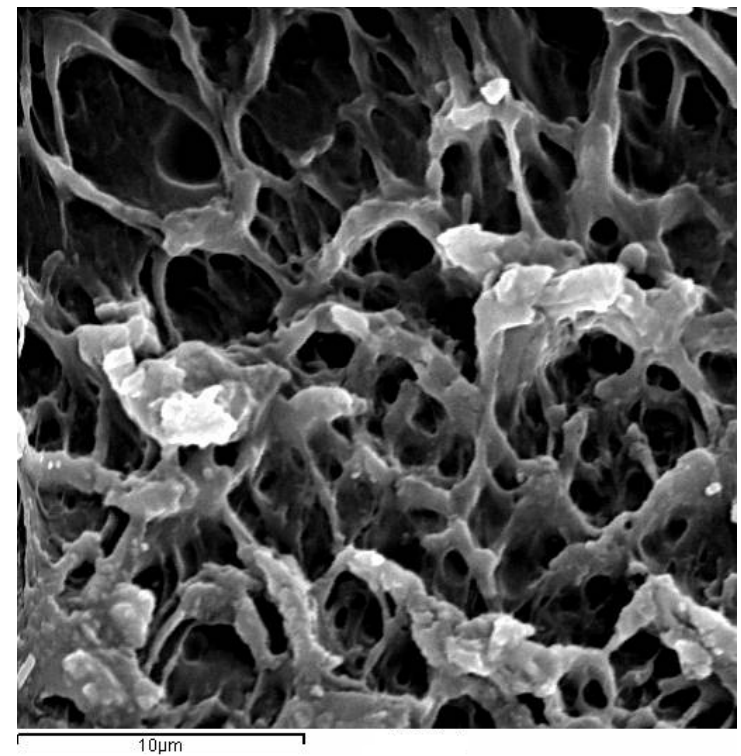

Figure 15. SEM micrograph of superabsorbent $(\times 4000)$ 


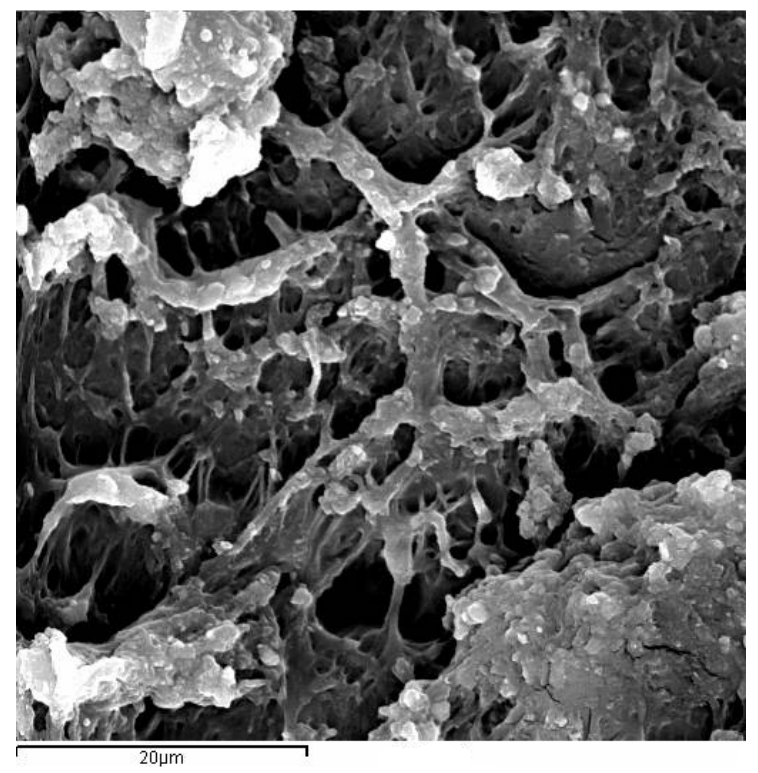

Figure 16. SEM micrograph of superabsorbent $(\times 2000)$

Figure 15 and Figure 16 are respectively the resin scanning photos magnified 4000 times and 2000 times. We can see that the product has already formed the uniform three-dimensional network structure. Compared with Figure 10 and Figure 11, the network structure is obviously dense and even. Microwave heating is used by dielectric loss principle, different from gradient transfer of the traditional heating method. Its internal and external synchronous heating causes product loose and porous. This is the reason why binary grafted amylum superabsorbent in microwave condition has better water absorption than that in traditional condition.

\subsection{Summary}

This chapter uses aqueous solution method for amylum grafted acrylic/acrylamide superabsorbent resin by microwave. Like traditional condition, polymerization temperature and polymerization time are fixed respectively. The chapter works out the best reaction condition of the preparation of corn amylum grafted acrylic/acrylamide superabsorbent resin is that reaction temperature is $35^{\circ} \mathrm{C}$ and reaction time is $1.5 \mathrm{~h}$. The absorbent ratio of water is up to 711 times and the absorbent ratio of physiological saline is up to 86 times.

The reaction time and temperature, the time of getting the product at the same bibulous rate by microwave is obviously slower than that using traditional method and the temperature is obviously lower. Under similar condition, the property of the product by microwave method is improved. What's more, reaction process doesn't need nitrogen protection under microwave condition. These phenomena explain that the synthesis of superabsorbent resin by means of microwave is of great value. 


\section{Exploration and research applications}

\subsection{Introduction}

Superabsorbent resin is commonly used in soil superabsorbent resins and soil ameliorators in agriculture and forestry gardening areas. A large amount researches at home and abroad have shown that the use of superabsorbent resin can significantly improve water content of soil, volume expansion rate, total porosity and capillary porosity and soil structure. It can also ease change of ground temperature, microbial activities and soil fertility. It always increase crop output, improve survival rate of trees and promote plant growth. In watershortage areas, the use of superabsorbent resin has significant water-saving effect.

China is listed as one of 13 short water countries and one of the world's most serious waterloss countries. The soil erosion areas are 3.67 million $\mathrm{km}^{2}$, which occupy $38.2 \%$ of the total land areas. The desertification lands are throughout 13 provinces, cities and autonomous regions of the country and inferior soil exists generally. The composition balance of organic and inorganic substances in soils was destroyed by the unrestrained using of chemical fertilizers. At the same time, our country is a populous country in the world and the implementation of the soil and water conservation is one of our basic policies as well as a systematic engineering under such serious circumstances. With the characteristic of big water absorbability and high water retention, applying superabsorbent resin to soil erosion control, desertification, agricultural water-saving is a very significant work.

Now this technology is not large-scale used for water-saving agriculture and the main reason as follows. Firstly, the price of superabsorbent resin is expensive and using in agriculture will increase the product cost. It is difficult to promote. Secondly, a lot of superabsorbent resin which theoretically has high bibulous rate and salt tolerance are in fact unsatisfactory when used in complex soil environment. Thirdly, farmers in some drought areas who are often poor and backward have no perceptual knowledge on superabsorbent resin and can't accept it. These points have decided that promoting superabsorbent resins application on agriculture really needs quite a long time. It requires researchers not only to development a new superabsorbent resin with good comprehensive performance, but also to find a multi-function superabsorbent resin which is drought resistant, pest resistant, fit for plant growth and not destroying the soil structure under the soil environment and considering the nutrition demands of plant growth.

This chapter has made a research on amylum superabsorbent grafted AA/AM under traditional condition and microwave condition. There own water retention and water absorption ability, water steam suppression, expansion effect and water retaining capacity in the soil was studied in this experiment, hoping can offer some help on the future application experiment in large scale.

\subsection{Experiment}

\subsubsection{Material and equipment}

- $\quad$ Amylum Superabsorbent Grafted AA/AM by Traditional Method (50 mesh) 
- $\quad$ Amylum Superabsorbent Grafted AA/AM by Microwave Method (50 mesh)

- Local Soil

- Commercial Grass Seed

- DZF-6021 Vacuum Drying Oven Shanghai Jinghong Experiment Instrument Co.LTD

\subsubsection{Experiment methods}

\subsubsection{Water retention of superabsorbent}

Taking bibulous saturated resin $50.0 \mathrm{~g}$ into Buchner funnel and putting a non-absorbent small board on it. Then putting weight on board, resting about 20 minute. The water mass was weight in different pressure.

\subsubsection{Pretreatment of experiment soil}

This study has selected the local loess as experiment object by air dry indoor and use drysievetest for pretreatment. Pat the soil slightly into small patch and dispose impurities. Then put the sample in $50^{\circ} \mathrm{C}$ oven and drought it out.

Grinding dried soil sample through a 50 mesh sieve and setting it in dry place for standby application.

\subsubsection{Bibulous rate of superabsorbent resin in soil}

Add superabsorbent resin of $0.00 \%, 0.10 \%, 0.15 \%, 0.20 \%, 0.25 \%, 0.30 \%$ into $15.0 \mathrm{~g}$ soil respectively. Mix them equally and pour them into tea bag. Put them into beakers and take them out after soaking, hanging them up until no water drop and weight them. Calculate bibulous rate of superabsorbent resin in soil according to equation 2.

$$
\mathrm{Q}=\left(\mathrm{W}_{1}-\mathrm{W}_{0}-\mathrm{W}_{2}\right) / \mathrm{W}_{2} \ldots
$$

Among:

- $\quad$ Q - bibulous rate of superabsorbent resin in soil

- $\quad \mathrm{W}_{0}$ - mass of water absorption of mixed soil /g

- W1- mass of water absorption of blank soil /g

- $\mathrm{W}_{2}$ - dosage of superabsorbent resin /g

\subsubsection{Experiment of soil spraying}

Taking five soils of $15.0 \mathrm{~g}$, adding superabsorbent resin of $0.00 \mathrm{~g}, 0.03 \mathrm{~g}, 0.06 \mathrm{~g}, 0.09 \mathrm{~g}, 0.12 \mathrm{~g}$ respectively, mixing equally and pouring them into glass tube, making a $1.0 \mathrm{~cm}$-diameter hole at the bottom of the glass tube and plugging the hole with cotton to prevent soil's leaking out. Spraying soil sample with $20.0 \mathrm{~mL}$ distilled water with the velocity of 5.0 $\mathrm{mL} / \mathrm{min}$ by separating funnel on the top of glass tube and the flow of water from the glass tube is caught by measuring cylinder of $20.0 \mathrm{~mL}$. The time and the water volume in the measuring cylinder was recorded when the water begins to flow out of the glass tube as soon as the flow stops. 


\subsubsection{Water steam suppression of soil}

Taking five Experiment soils of $20.0 \mathrm{~g}$ into five same beakers respectively, adding superabsorbent resin of $0.00 \%, 0.15 \%, 0.30 \%, 0.45 \%, 0.60 \%$ of soil dosage, mixing well, joining with de-ionized water of $10.0 \mathrm{~g}$ and weighing it until the soil fully wet. Put soil sample into a $35^{\circ} \mathrm{C}$ oven to dry it and weigh it every $30 \mathrm{~min}$. Drawing curve by evaporation time and soil moisture and investigating effect of water steam suppression of soil. Calculating water content in soil according to equation 3:

$$
\mathrm{W} \%=\left(\mathrm{M}_{1}-\mathrm{M}_{2}\right) / \mathrm{M}_{1} \times 100 \% \ldots
$$

Among:

- $\quad \mathrm{M}_{1}$ - initial mass /g

- $\quad M_{2}$ - present mass /g

\subsubsection{Expansion effect of superabsorbent resin}

Taking five soils of $20.0 \mathrm{~g}$, adding superabsorbent resin of $0.00 \mathrm{~g}, 0.03 \mathrm{~g}, 0.06 \mathrm{~g}, 0.09 \mathrm{~g}$, $0.12 \mathrm{~g}$ respectively, mixing equally, measuring $15.0 \mathrm{~mL}$ mixed soil using measuring cylinder of $25.0 \mathrm{~mL}$, adding enough distilled water, removing extra water by burette and measuring the volume of moisture soil samples. Calculating expansion rate according to equation 4 :

$$
\mathrm{V} \%=(\mathrm{V}-15) / 15 \times 100 \%
$$

Among:

- V - Volume of wet soil sample /mL

\subsubsection{Experiment of grass planting}

Three experiment soils of $200.0 \mathrm{~g}$ were putted into three breathable flowerpots, adding $1.0 \mathrm{~g}$ superabsorbent resin prepared by traditional method in the first one, adding $1.0 \mathrm{~g}$ superabsorbent resin prepared by microwave method in the second one and the last one acts as blank soil. Choosing 30 plump grass seeds into three flowerpots averagely, adding water of $150.0 \mathrm{~mL}$ respectively, watering $10.0 \mathrm{~mL}$ every day and ensuring the sun exposure time, watering frequency and water dosage of three flowerpots are same. Observing them for 20 days and taking notes of grass growing condition. Planting test room temperature is kept $15-25^{\circ} \mathrm{C}$. Planting irrigation water is local tap water.

\subsection{Results and discussion}

\subsubsection{Resin self-protection water}

Two resins both have good performance of water retention and products by microwave method are slightly better than that by traditional method from the Figure 17. 


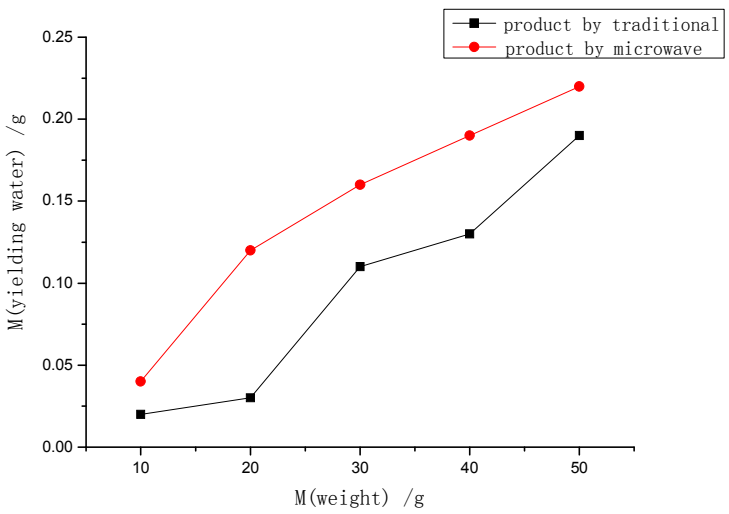

Figure 17. Test of water retention

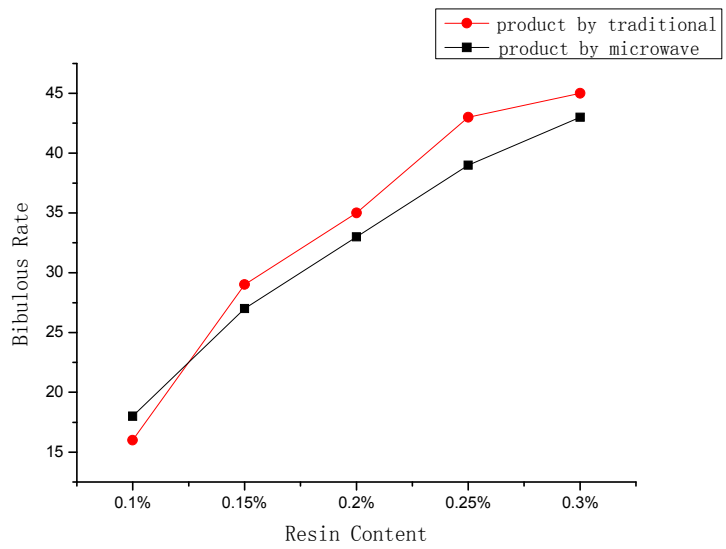

Figure 18. Bibulous rate of superabsorbent resin in soil 


\subsubsection{Superabsorbent resin in soil}

We have found that with the increase of the dosage of resin, bibulous rate increases. This is because that with the increase of resin content in soil, the water absorption of resin is more obvious than water absorption of soil itself. Water absorption capacity of soil with superabsorbent resin is less than that without superabsorbent resin. This is because superabsorbent resin mixed in soil is influenced by soil oppression and free metal ions in the process of water swelling. (Figure 18)

The performance of products by microwave method is obviously better than that by traditional method. So products by microwave method are chosen for the following experiments.

\subsubsection{Experiment result of soil spraying}

The main purpose of soil spraying experiment is to test whether superabsorbent resin can form a water-resisting layer in soil of certain depth to prevent soil erosion in rainfall or irrigation cases and whether it can absorb a lot of water storing for use in drought.

From Table 3, we find that after adding superabsorbent resin, the water outflow decreases, so water retention ability of soil strengthens. We also see that after joining with superabsorbent resin, speed of water leakage has decelerated. It is because that the resin has crosslinked with its surrounding soil particles and formed a water-resisting layer, so it can prevent moisture infiltration. Therefore, water retention ability is stronger as less water flow.

It shows that soil mixed with superabsorbent resin can clearly improve the performance of water retention.

\begin{tabular}{|c|c|c|c|c|c|}
\hline & $0.00 \mathrm{~g}$ & $0.03 \mathrm{~g}$ & $0.06 \mathrm{~g}$ & $0.09 \mathrm{~g}$ & $0.12 \mathrm{~g}$ \\
\hline VWater Spraying & $20 \mathrm{~mL}$ & $20 \mathrm{~mL}$ & $20 \mathrm{~mL}$ & $20 \mathrm{~mL}$ & $20 \mathrm{~mL}$ \\
\hline V Spraying & $5 \mathrm{~mL} / \mathrm{min}$ & $5 \mathrm{~mL} / \mathrm{min}$ & $5 \mathrm{~mL} / \mathrm{min}$ & $5 \mathrm{~mL} / \mathrm{min}$ & $5 \mathrm{~mL} / \mathrm{min}$ \\
\hline to & $17 \mathrm{~min}$ & $21 \mathrm{~min}$ & $26 \mathrm{~min}$ & $35 \mathrm{~min}$ & $38 \mathrm{~min}$ \\
\hline VYielding Water & $9.5 \mathrm{~mL}$ & $8.4 \mathrm{~mL}$ & $6.9 \mathrm{~mL}$ & $6.1 \mathrm{~mL}$ & $5.8 \mathrm{~mL}$ \\
\hline
\end{tabular}

Table 3. Experiment result of soil spraying

\subsubsection{Water steam suppression of soil}

Superabsorbent resin has strong performance of water retention, even in the condition of pressure and heating. This experiment shows that different dosages of superabsorbent resin have inhibiting effects on water evaporation under $35^{\circ} \mathrm{C}$. Figure 19 show that soil without resin loses water faster when is heated. Water content is already $0.5 \%$ at 180 minute and the water in soil sample has nearly evaporated out. The water content of soil samples being added with resin of $0.15 \%, 0.3 \%, 0.45 \%, 0.6 \%$ are $2.3 \%, 3.3 \%, 3.5 \%$ and $4.1 \%$.Obviously speed of water evaporation in soil has been inhibited by resin. With the increase of the resin content, inhibition of evaporation also increases. 


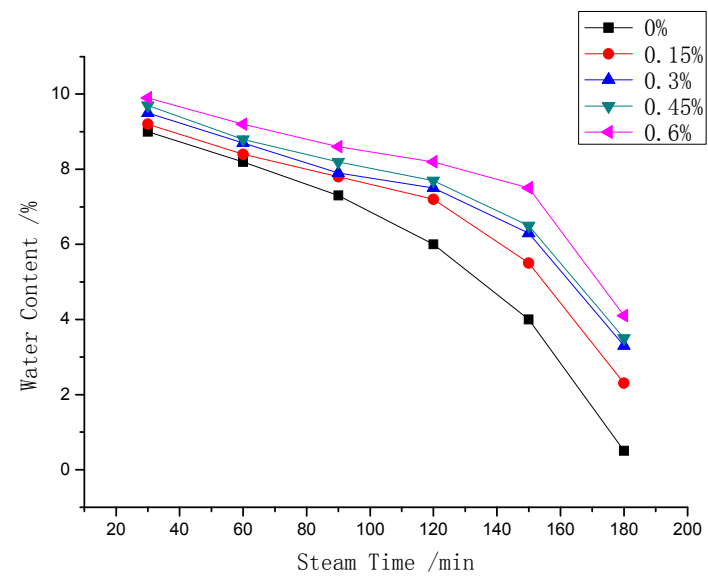

Figure 19. Effect of water steam suppression of soil

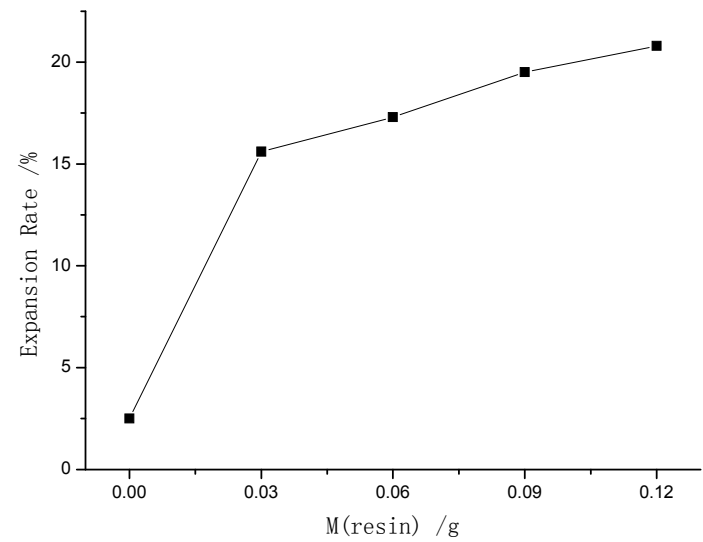

Figure 20. Effect of expansion effect of resin 


\subsubsection{Expansion effect of superabsorbent resin}

The volume of soil has significantly increased after adding resin. The volume inflation rate has been amount to $20.8 \%$ when we add $0.12 \mathrm{~g}$ resin in $20.0 \mathrm{~g}$ soil. The research has found that the volume inflation rate increases with the increasing use of resin. This expansion effect is very good for improving soil compactness, regulating gas permeability of soil and making the soil structure favorable (Figure 20).

\subsubsection{Experiment result of grass planting}

In this experiment, we have not found that superabsorbent resin can fight for water with plants so that influence plant growth. The suitable dosage of superabsorbent resin has certainly facilitated plant growth and grass without resin is significantly weaker growth than grass with resin. They all have significantly effects with resin added either from germination rate or from average height (Table 4).

\begin{tabular}{|c|c|c|c|c|c|}
\hline Type & Days & Seed Number & Germinative Number & Average Height/cm & Plant Growth \\
\hline 1 & 20 & 10 & 8 & 51.12 & Bloom,Thick Stem \\
\hline 2 & 20 & 10 & 7 & 50.07 & Bloom, Thin Stem \\
\hline 3 & 20 & 10 & 3 & 29.87 & Poor, Thin Stem \\
\hline
\end{tabular}

Table 4. Experiment result of grass planting

\subsection{Summary}

Through testing water retention of resin, bibulous rate in soil, experiment of soil spraying, water steam suppression of soil, expansion effect of superabsorbent resin and experiment of grass planting, a simple research on application of superabsorbent resin has been discussed. The results show that: soils dashed with superabsorbent resin have improved the water retention of soil and soil compactness, regulate gas permeability of soil and make the soil structure favorable. The suitable dosage of superabsorbent resin has certainly facilitated plant growth.

\section{Author details}

Hao Ren, Zhen Niu, Juan Wang and Jing Ning

Shandong Provincial Key Laboratory of Fluorine Chemistry and Chemical Materials, School of Chemistry and Chemical Engineering, University of Jinan, Shandong, China

\section{Acknowledgement}

This work was financially supported by the Publishing Fundation Project of University of Jinan. Special thanks are given to the research team for providing support in this study. 


\section{References}

Du T S \& Kang S Z. (2000). Situation and expectation of application of water-saving agent on agriculture. Research on Agricultural Modernization, 2000, 21(5):317-320.

Fushan Chen \& Qian Gong. (2007). Influential Factors and Optimization of Synthesis of Cassava Amylum Superabsorbent Grafted AA/AM. Leather Chemical Industry, 2007, 24(5):38-42.

Ge Y X \& Zhang B Z. (2006). Preparation and Characterization of Superabsorbent Resin. Science and Technology Journal of Hebei University, 2006, 27(4):285-287.

Hongke Tang \& Qi Chen. (2007). Synthesis of Amylum Superabsorbent Grafted AA/AM. Synthesis Chemistry, 2007, 15(5):643-646.

Kabiri K \& Zohuriaan-Mehr M J. (2003). Superabsorbent Hydrogel Composites. Polymers for Advanced Technologies, 2003, 14 (6):438-444.

Kabiri K, Omidian H \& Zohuriaan-Mehr M J. (2003). Novel Approach to Highly Porous Superabsorbent Hydrogels: Synergistic Effect of Porogens on Porosity and Swelling Rate. Polymer International, 2003, 52(7):1158-1164.

Lokhande H T \& Lyer V. (1992). Water-Superabsorbent Polymers through Gamma Radiation-Induced Graft-Copolymerization of Acrylontirile on Guar gum. Journal of Applied Polymer Science, 1992, 45:2031-2036.

Liu Z S \& Rempel G L. (1997). Preparation of Superabsorbent Polymers by Crosslinking Acrylic Acid and Acrylamide Copolymers. Journal of Applied Polymer Science, 1997, 64 (7):1345-1353.

Lu S J, Duan M L \& Lin S B. (2003). Synthesis of Superabsorbent Starch-Graft-Poly (potassium acrylate-co-acrylamide) and Its Properties. Journal of Applied Polymer Science, 2003, 88 (6):1536-1542.

Li D L \& Yang P L. (2004). Experimental Studies on Water-saving Agent in Grape Cultivation. Agricultural engineering journal, 2004, 20(3): 51-54.

Pourjavadi A, Mahdavinia G R \& Zohuriaan-Mehr M J. (2003). Modified Chitosan II HchitoPAN, A Novel pH-responsive Superabsorbent Hydrogel. Journal of Applied Polymer Science, 2003, 90 (11):3115-3121.

Qinhan Jin. (1999). Microwave Chemistry. Beijing. Science Press, 1999.

Raju K M \& Raju M P. (2001). Synthesis and Swelling Properties of Superabsorbent Copolymers. Advances in Polymer Technology.2001, 20(2):146-154.

Shouyong Fan. (2003). Microwave Technology and Microwave Circuit. Beijing. Mechanical Industry Press, 2003.

Suda K, Mongkolsawat K \& Sonsuk M. (2002). Synthesis and property characterization of cassava starch grafted poly [acrylamide-co-(maleic acid)] superabsorbent via girradiation. Polymer, 2002, 43(14): 3915-3924.

Taden A, Tait A H \& Kraft A. (2002). Synthesis and Polymerization of 5-(Methacrylamido) tatrazole, a Water-soluble Acidic Monomer. Journal of Polymer Science, 2002, 40 (23):43334343.

Wangxi Zhang. (2004). Application of Microwave in Chemical Reaction. Synthesis technology and application, 2004, 19(3):25-27. 
Yan Huang \& Jiarui Shen. (1995). Research on Thickening Ability of Amylum Grafted Acrylamide. Polymer Materials Science and Engineering, 1995, 11(3):98-102.

Yan Q Z \& Su X T. (2005). Wave Synthesis of Cassava Amylum Superabsorbent Grafted AA/AM. Chem J Chin Univ, 2005, 26(7):1363-1365.

Zhou M \& Lin J M. (2000). Research on Amylum Grafted Acrylamide. Journal of Overseas University (Natural Science), 2000, 20 (4):362-365. 